\title{
ALUMINUM PHOSPHIDE POISONING
}

Sir,

Aluminum phosphide poisoning is an important medical emergency which physicians in the developing countries, including India, deal with very commonly. While the authors have commented on the effects of various clinical and biochemical parameters on outcome, some other factors might have played a role in determining the prognosis. ${ }^{[1]}$

It would be of interest to know if the tablets were fresh or exposed, since exposed tablets lose their lethality and may cause less severe manifestations. Administration of magnesium sulfate has been shown to improve outcome, possibly by dual action of membrane stabilization and correction of hypomagnese mia. ${ }^{[2]}$ Since only 9 out of the 49 patients received magnesium sulfate, it will be 
interesting to know the dosage used and if the administration of magnesium sulfate made any difference to the outcome.

Also, the authors have commented on the presence of myocardial ischemia. It would be of interest to know how this was diagnosed, since ECG changes of severe toxic myocarditis as seen in aluminum phosphide poisoning can easily mimic those of ischemia. ${ }^{[3]}$ Probably the changes authors have mentioned are a result of severe myocarditis rather than ischemia per se. It would also be of interest to know the number of patients who had altered sensorium and the cause of altered sensorium.

VISHAL SHARMA, ALKA SHARMA

Department of Medicine University College of Medical Sciences, Delhi, India.

Correspondence: Dr. Vishal Sharma, University College of Medical Sciences, Delhi, India E-mail: docvishalsharma@gmail.com

\section{REFERENCES}

1. Louriz M, Dendane T, Abidi K, Madani N, Abouqal $R$, Zeggwagh AA. Prognostic factors of acute aluminum phosphide poisoning. Indian J Med Sci 2009;63:227-34.

2. Chugh SN, Kumar P, Aggarwal HK, Sharma A, Mahajan SK, Malhotra KC. Efficacy of magnesium sulphate in aluminium phosphide poisoning-comparison of two different dose schedules. J Assoc Physicians India 1994;42:373-5.

3. Soltaninejad K, Shadnia S, Ziyapour B, Brent J. Aluminum phosphide intoxication mimicking ischemic heart disease led to unjustified treatment with streptokinase. Clin Toxicol (Phila) 2009;47:908-9.

DOI: 10.4103/0019-5359.59991 